\title{
New Classes of Analytic Functions Involving Generalized Noor Integral Operator
}

\author{
Rabha W. Ibrahim and Maslina Darus \\ School of Mathematical Sciences, Faculty of Science and Technology, Universiti Kebangsaan Malaysia, \\ Selangor Darul Ehsan, Bangi 43600, Malaysia
}

Correspondence should be addressed to Maslina Darus, maslina@pkrisc.cc.ukm.my

Received 22 March 2008; Accepted 25 April 2008

Recommended by Jozsef Szabados

The present article investigates new classes of functions involving generalized Noor integral operator. Some properties of these functions are studied including characterization and distortion theorems. Moreover, we illustrate sufficient conditions for subordination and superordination for analytic functions.

Copyright $(2008$ R. W. Ibrahim and M. Darus. This is an open access article distributed under the Creative Commons Attribution License, which permits unrestricted use, distribution, and reproduction in any medium, provided the original work is properly cited.

\section{Introduction and preliminaries}

Let $\mathscr{H}$ be the class of functions analytic in $U$ and let $\mathscr{H}[a, n]$ be the subclass of $\mathscr{H}$ consisting of functions of the form $f(z)=a+a_{n} z^{n}+a_{n+1} z^{n+1}+\cdots$. Let $\mathscr{A}$ be the subclass of $\mathscr{H}$ consisting of functions of the form $f(z)=z+a_{2} z^{2}+\cdots$.

Denote by $D^{\alpha}: \mathcal{A} \rightarrow \mathcal{A}$ the operator defined by

$$
D^{\alpha}:=\frac{z}{(1-z)^{\alpha+1}} * f(z), \quad \alpha>-1,
$$

where $(*)$ refers to the Hadamard product or convolution. Then implies that

$$
D^{n} f(z)=\frac{z\left(z^{n-1} f(z)\right)^{(n)}}{n !}, \quad n \in N_{0}=N \cup\{0\} \text {. }
$$

We note that $D^{0} f(z)=f(z)$ and $D^{\prime} f(z)=z f^{\prime}(z)$. The operator $D^{n} f$ is called Ruscheweyh derivative of $n$th order of $f$. Noor $[1,2]$ defined and studied an integral operator $I_{n}: \mathcal{A} \rightarrow \mathcal{A}$ analogous to $D^{n} f$ as follows. 
Let $f_{n}(z)=z /(1-z)^{n+1}, n \in N_{0}$, and let $f_{n}^{(-1)}$ be defined such that

$$
f_{n}(z) * f_{n}^{(-1)}(z)=\frac{z}{1-z}
$$

Then

$$
I_{n} f(z)=f_{n}^{(-1)}(z) * f(z)=\left[\frac{z}{(1-z)^{n+1}}\right]^{(-1)} * f(z) .
$$

Note that $I_{0} f(z)=z f^{\prime}(z)$ and $I_{1} f(z)=f(z)$. The operator $I_{n}$ is called the Noor Integral of $n$th order of $f$. Using (1.3), (1.4), and a well-known identity for $D^{n} f$, we have

$$
(n+1) I_{n} f(z)-n I_{n+1} f(z)=z\left(I_{n+1} f(z)\right)^{\prime} .
$$

Using hypergeometric functions ${ }_{2} F_{1},(1.4)$ becomes

$$
I_{n} f(z)=\left[z_{2} F_{1}(1,1 ; n+1, z)\right] * f(z),
$$

where ${ }_{2} F_{1}(a, b ; c, z)$ is defined by

$$
{ }_{2} F_{1}(a, b ; c, z)=1+\frac{a b}{c} \frac{z}{1 !}+\frac{a(a+1) b(b+1)}{c(c+1)} \frac{z^{2}}{2 !}+\cdots .
$$

For complex parameters

$$
\begin{aligned}
& \alpha_{1}, \ldots, \alpha_{q}\left(\frac{\alpha_{j}}{A_{j}} \neq 0,-1,-2, \ldots ; j=1, \ldots, q\right), \\
& \beta_{1}, \ldots, \beta_{p}\left(\frac{\beta_{j}}{B_{j}} \neq 0,-1,-2, \ldots ; j=1, \ldots, p\right),
\end{aligned}
$$

the Fox-Wright generalization $\Psi_{p}[z]$ of the hypergeometric ${ }_{q} F_{p}$ function by(see [3-5])

$$
\begin{aligned}
{ }_{q} \Psi_{p}\left[\begin{array}{c}
\left(\alpha_{1}, A_{1}\right), \ldots,\left(\alpha_{q}, A_{q}\right) ; \\
\left(\beta_{1}, B_{1}\right), \ldots,\left(\beta_{p}, B_{p}\right) ;
\end{array}\right] & ={ }_{q} \Psi_{p}\left[\left(\alpha_{j}, A_{j}\right)_{1, q} ;\left(\beta_{j}, B_{j}\right)_{1, p} ; z\right] \\
& :=\sum_{n=0}^{\infty} \frac{\Gamma\left(\alpha_{1}+n A_{1}\right) \cdots \Gamma\left(\alpha_{q}+n A_{q}\right)}{\Gamma\left(\beta_{1}+n B_{1}\right) \cdots \Gamma\left(\beta_{p}+n B_{p}\right)} \frac{z^{n}}{n !} \\
& =\sum_{n=0}^{\infty} \frac{\prod_{j=1}^{q} \Gamma\left(\alpha_{j}+n A_{j}\right)}{\prod_{j=1}^{p}\left(\beta_{j}+n B_{j}\right)} \frac{z^{n}}{n !},
\end{aligned}
$$

where $A_{j}>0$ for all $j=1, \ldots, q, B_{j}>0$ for all $j=1, \ldots, p$, and $1+\sum_{j=1}^{p} B_{j}-\sum_{j=1}^{q} A_{j} \geq 0$ for suitable values $|z|$. For special case, when $A_{j}=1$ for all $j=1, \ldots, q$, and $B_{j}=1$ for all $j=1, \ldots, p$, we have the following relationship:

$$
\begin{gathered}
{ }_{q} F_{p}\left(\alpha_{1}, \ldots, \alpha_{q} ; \beta_{1}, \ldots, \beta_{p} ; z\right)=\Omega_{q} \Psi_{p}\left[\left(\alpha_{j}, 1\right)_{1, q} ;\left(\beta_{j}, 1\right)_{1, p} ; z\right], \\
q \leq p+1 ; \quad q, p \in \mathbb{N}_{0}=\mathbb{N} \cup\{0\}, \quad z \in U,
\end{gathered}
$$


where

$$
\Omega:=\frac{\Gamma\left(\beta_{1}\right) \cdots \Gamma\left(\beta_{p}\right)}{\Gamma\left(\alpha_{1}\right) \cdots \Gamma\left(\alpha_{q}\right)}
$$

We introduce a function $\left(z_{q} \Psi_{p}\left[\left(\alpha_{j}, A_{j}\right)_{1, q} ;\left(\beta_{j}, B_{j}\right)_{1, p} ; z\right]\right)^{-1}$ given by

$$
\begin{gathered}
\left(z_{q} \Psi_{p}\left[\left(\alpha_{j}, A_{j}\right)_{1, q^{\prime}}\left(\beta_{j}, B_{j}\right)_{1, p^{\prime}} z\right]\right) *\left(z_{q} \Psi_{p}\left[\left(\alpha_{j}, A_{j}\right)_{1, q^{\prime}}\left(\beta_{j}, B_{j}\right)_{1, p^{\prime}} z\right]\right)^{-1} \\
=\frac{z}{(1-z)^{\lambda+1}}=z+\sum_{n=2}^{\infty} \frac{(\lambda+1)_{n-1}}{(n-1) !} z^{n}, \quad(\lambda>-1)
\end{gathered}
$$

and obtain the following linear operator:

$$
I_{\lambda}\left[\left(\alpha_{j}, A_{j}\right)_{1, q} ;\left(\beta_{j}, B_{j}\right)_{1, p}\right] f(z)=\left(z_{q} \Psi_{p}\left[\left(\alpha_{j}, A_{j}\right)_{1, q} ;\left(\beta_{j}, B_{j}\right)_{1, p} ; z\right]\right)^{-1} * f(z),
$$

where $f \in \mathcal{A}, z \in U$, and

$$
\left(z_{q} \Psi_{p}\left[\left(\alpha_{j}, A_{j}\right)_{1, q} ;\left(\beta_{j}, B_{j}\right)_{1, p} ; z\right]\right)^{-1}=z+\sum_{n=2}^{\infty} \frac{\prod_{j=1}^{p} \Gamma\left(\beta_{j}+(n-1) B_{j}\right)}{\prod_{j=1}^{q} \Gamma\left(\alpha_{j}+(n-1) A_{j}\right)}(\lambda+1)_{n-1} z^{n}
$$

For some computation, we have

$$
I_{\lambda}\left[\left(\alpha_{j}, A_{j}\right)_{1, q} ;\left(\beta_{j}, B_{j}\right)_{1, p}\right] f(z)=z+\sum_{n=2}^{\infty} \frac{\prod_{j=1}^{p} \Gamma\left(\beta_{j}+(n-1) B_{j}\right)}{\prod_{j=1}^{q} \Gamma\left(\alpha_{j}+(n-1) A_{j}\right)}(\lambda+1)_{n-1} a_{n} z^{n},
$$

where $(a)_{n}$ is the Pochhammer symbol defined by

$$
(a)_{n}=\frac{\Gamma(a+n)}{\Gamma(a)}= \begin{cases}1, & n=0 \\ a(a+1) \cdots(a+n-1), & n=\{1,2, \ldots\}\end{cases}
$$

From (1.15) we have the following result.

Lemma 1.1. Let $f(z) \in \mathcal{A}$ for all $z \in U$ then

(i) $I_{0}\left[(1,1)_{1,1} ;(1,1 /(n-1))_{1, p}\right] f(z)=f(z)$.

(ii) $I_{1}\left[(1,1)_{1,1} ;(1,1 /(n-1))_{1, p}\right] f(z)=z f^{\prime}(z)$.

(iii) $z\left[I_{\lambda}\left[\left(\alpha_{j}, A_{j}\right)_{1, q} ;\left(\beta_{j}, B_{j}\right)_{1, p}\right] f(z)\right]^{\prime}=(\lambda+1) I_{\lambda+1}\left[\left(\alpha_{j}, A_{j}\right)_{1, q} ;\left(\beta_{j}, B_{j}\right)_{1, p}\right] f(z)-\lambda I_{\lambda}\left[\left(\alpha_{j}, A_{j}\right)_{1, q}\right.$; $\left.\left(\beta_{j}, B_{j}\right)_{1, p}\right] f(z)$.

In the following definitions, we introduce new classes of analytic functions containing generalized Noor integral operator (1.15). 
Definition 1.2. Let $f(z) \in \mathcal{A}$ then $f(z) \in S_{\lambda}^{\mu}\left[\left(\alpha_{j}, A_{j}\right)_{1, q} ;\left(\beta_{j}, B_{j}\right)_{1, p}\right]$ if and only if

$$
\mathfrak{R}\left\{\frac{z\left[I_{\lambda}\left[\left(\alpha_{j}, A_{j}\right)_{1, q^{\prime}}\left(\beta_{j}, B_{j}\right)_{1, p}\right] f(z)\right]^{\prime}}{I_{\lambda}\left[\left(\alpha_{j}, A_{j}\right)_{1, q} ;\left(\beta_{j}, B_{j}\right)_{1, p}\right] f(z)}\right\}>\mu, \quad 0 \leq \mu<1, \quad z \in U .
$$

Definition 1.3. Let $f(z) \in \mathcal{A}$ then $f(z) \in C_{\lambda}^{\mu}\left[\left(\alpha_{j}, A_{j}\right)_{1, q} ;\left(\beta_{j}, B_{j}\right)_{1, p}\right]$ if and only if

$$
\mathfrak{R}\left\{\frac{\left(z\left[I_{\lambda}\left[\left(\alpha_{j}, A_{j}\right)_{1, q^{\prime}} ;\left(\beta_{j}, B_{j}\right)_{1, p}\right] f(z)\right]^{\prime}\right)^{\prime}}{\left(I_{\lambda}\left[\left(\alpha_{j}, A_{j}\right)_{1, q^{\prime}} ;\left(\beta_{j}, B_{j}\right)_{1, p}\right] f(z)\right)^{\prime}}\right\}>\mu, \quad 0 \leq \mu<1, \quad z \in U .
$$

Let $F$ and $G$ be analytic functions in the unit disk $U$. The function $F$ is subordinate to $G$, written $F \prec G$, if $G$ is univalent, $F(0)=G(0)$ and $F(U) \subset G(U)$. Or given two functions $F(z)$ and $G(z)$, which are analytic in $U$, the function $F(z)$ is said to be subordination to $G(z)$ in $U$ if there exists a function $h(z)$, analytic in $U$ with

$$
h(0)=0, \quad|h(z)|<1 \quad \forall z \in U,
$$

such that

$$
F(z)=G(h(z)) \quad \forall z \in U
$$

Let $\phi: \mathbb{C}^{2} \rightarrow \mathbb{C}$ and let $h$ be univalent in $U$. If $p$ is analytic in $U$ and satisfies the differential subordination $\left.\phi(p(z)), z p^{\prime}(z)\right) \prec h(z)$ then $p$ is called a solution of the differential subordination. The univalent function $q$ is called a dominant of the solutions of the differential subordination, $p \prec q$. If $p$ and $\left.\phi(p(z)), z p^{\prime}(z)\right)$ are univalent in $U$ and satisfy the differential superordination $\left.h(z) \prec \phi(p(z)), z p^{\prime}(z)\right)$ then $p$ is called a solution of the differential superordination. An analytic function $q$ is called subordinant of the solution of the differential superordination if $q \prec p$. Let $\Phi$ be an analytic function in a domain containing $f(U), \Phi(0)=0$ and $\Phi^{\prime}(0)>0$.

The function $f \in \mathcal{A}$ is called $\Phi$-like if

$$
\mathfrak{R}\left\{\frac{z f^{\prime}(z)}{\Phi(f(z))}\right\}>0, \quad z \in U
$$

This concept was introduced by Brickman [6] and established that a function $f \in \mathcal{A}$ is univalent if and only if $f$ is $\Phi$-like for some $\Phi$.

Definition 1.4. Let $\Phi$ be analytic function in a domain containing $f(U), \Phi(0)=0, \Phi^{\prime}(0)=1$, and $\Phi(\omega) \neq 0$ for $\omega \in f(U)-0$. Let $q(z)$ be a fixed analytic function in $U, q(0)=1$. The function $f \in \mathcal{A}$ is called $\Phi$-like with respect to $q$ if

$$
\frac{z f^{\prime}(z)}{\Phi(f(z))} \prec q(z), \quad z \in U
$$


In the present paper, we apply a method based on the differential subordination in order to obtain subordination results involving generalized Noor integral operator for a normalized analytic function $f(z) z \in U$

$$
q_{1}(z) \prec \frac{z\left[I_{\curlywedge}\left[\left(\alpha_{j}, A_{j}\right)_{1, q} ;\left(\beta_{j}, B_{j}\right)_{1, p}\right] f(z)\right]^{\prime}}{\Phi\left[I_{\curlywedge}\left[\left(\alpha_{j}, A_{j}\right)_{1, q} ;\left(\beta_{j}, B_{j}\right)_{1, p}\right] f(z)\right]} \prec q_{2}(z) .
$$

In order to prove our subordination and superordination results, we need to the following lemmas in the sequel.

Definition 1.5 (see [7]). Denote by $Q$ the set of all functions $f(z)$ that are analytic and injective on $\bar{U}-E(f)$, where $E(f):=\left\{\zeta \in \partial U: \lim _{z \rightarrow \zeta} f(z)=\infty\right\}$ and are such that $f^{\prime}(\zeta) \neq 0$ for $\zeta \in$ $\partial U-E(f)$.

Lemma 1.6 (see [8]). Let $q(z)$ be univalent in the unit disk $U$ and $\theta$ and let $\phi$ be analytic in a domain $D$ containing $q(U)$ with $\phi(w) \neq 0$, when $w \in q(U)$. Set $Q(z):=z q^{\prime}(z) \phi(q(z)), h(z):=$ $\theta(q(z))+Q(z)$. Suppose that

(1) $Q(z)$ is starlike univalent in $U$,

(2) $\mathfrak{R}\left(z h^{\prime}(z) / Q(z)\right)>0$ for $z \in U$.

If

$$
\theta(p(z))+z p^{\prime}(z) \phi(p(z)) \prec \theta(q(z))+z q^{\prime}(z) \phi(q(z))
$$

then

$$
p(z) \prec q(z)
$$

and $q(z)$ is the best dominant.

Lemma 1.7 ([9]). Let $q(z)$ be convex univalent in the unit disk $U$ and let $\vartheta$ and $\varphi$ be analytic in a domain $D$ containing $q(U)$. Suppose that

(1) $z q^{\prime}(z) \varphi(q(z))$ is starlike univalent in $U$,

(2) $\Re\left\{\vartheta^{\prime}(q(z)) / \varphi(q(z))\right\}>0$ for $z \in U$.

If $p(z) \in \mathscr{H}[q(0), 1] \cap Q$, with $p(U) \subseteq D$ and $\vartheta(p(z))+z p^{\prime}(z) \varphi(z)$ being univalent in $U$ and

$$
\vartheta(q(z))+z q^{\prime}(z) \varphi(q(z)) \prec \vartheta(p(z))+z p^{\prime}(z) \varphi(p(z))
$$

then

$$
q(z) \prec p(z)
$$

and $q(z)$ is the best subordinant. 


\section{Characterization properties and distortion theorems}

In this section, we investigate the characterization properties for the function $f(z) \in \mathcal{A}$ to belong to the classes $S_{\lambda}^{\mu}\left[\left(\alpha_{j}, A_{j}\right)_{1, q} ;\left(\beta_{j}, B_{j}\right)_{1, p}\right]$ and $C_{\lambda}^{\mu}\left[\left(\alpha_{j}, A_{j}\right)_{1, q} ;\left(\beta_{j}, B_{j}\right)_{1, p}\right]$ by obtaining the coefficient bounds. Further, we prove the distortion theorems when $f(z) \in S_{\lambda}^{\mu}\left[\left(\alpha_{j}\right.\right.$, $\left.\left.A_{j}\right)_{1, q} ;\left(\beta_{j}, B_{j}\right)_{1, p}\right]$ and $f(z) \in C_{\lambda}^{\mu}\left[\left(\alpha_{j}, A_{j}\right)_{1, q} ;\left(\beta_{j}, B_{j}\right)_{1, p}\right]$.

Theorem 2.1. Let $f(z) \in \mathcal{A}$. Then $f(z) \in S_{\lambda}^{\mu}\left[\left(\alpha_{j}, A_{j}\right)_{1, q} ;\left(\beta_{j}, B_{j}\right)_{1, p}\right]$ if and only if

$$
\sum_{n=2}^{\infty} H_{n-1}\left|a_{n}\right|\left|\mu(\lambda+1)_{n-1}-\left((\lambda+1)_{n}-(\lambda)_{n}\right)\right| \leq 1-\mu, \quad 0 \leq \mu<1,
$$

where

$$
H_{n-1}:=\frac{\prod_{j=1}^{p} \Gamma\left(\beta_{j}+(n-1) B_{j}\right)}{\prod_{j=1}^{q} \Gamma\left(\alpha_{j}+(n-1) A_{j}\right)} .
$$

Proof. Suppose that (2.1) holds. Then by using Lemma 1.1 and for $z \in U$, we have

$$
\begin{aligned}
\mathfrak{R}\left\{\frac{z\left[I_{\lambda}\left[\left(\alpha_{j}, A_{j}\right)_{1, q^{\prime}}\left(\beta_{j}, B_{j}\right)_{1, p}\right] f(z)\right]^{\prime}}{I_{\lambda}\left[\left(\alpha_{j}, A_{j}\right)_{1, q^{\prime}}\left(\beta_{j}, B_{j}\right)_{1, p}\right] f(z)}\right\} & \leq\left|\frac{z\left[I_{\lambda}\left[\left(\alpha_{j}, A_{j}\right)_{1, q^{\prime}}\left(\beta_{j}, B_{j}\right)_{1, p}\right] f(z)\right]^{\prime}}{I_{\lambda}\left[\left(\alpha_{j}, A_{j}\right)_{1, q^{\prime}}\left(\beta_{j}, B_{j}\right)_{1, p}\right] f(z)}\right| \\
& \leq \frac{1+\sum_{n=2}^{\infty} H_{n-1}\left|a_{n}\right|\left((\lambda+1)_{n}-(\lambda)_{n}\right)}{1+\sum_{n=2}^{\infty} H_{n-1}\left|a_{n}\right|(\lambda+1)_{n-1}} .
\end{aligned}
$$

This last expression is greater than $\mu$, if (2.1) holds this implies that $f(z) \in S_{\lambda}^{\mu}\left[\left(\alpha_{j}\right.\right.$, $\left.\left.A_{j}\right)_{1, q} ;\left(\beta_{j}, B_{j}\right)_{1, p}\right]$. On the other hand, assume that $f(z) \in S_{\lambda}^{\mu}\left[\left(\alpha_{j}, A_{j}\right)_{1, q} ;\left(\beta_{j}, B_{j}\right)_{1, p}\right]$ then

$$
\mathfrak{R}\left\{\frac{z\left[I_{\lambda}\left[\left(\alpha_{j}, A_{j}\right)_{1, q^{\prime}}\left(\beta_{j}, B_{j}\right)_{1, p}\right] f(z)\right]^{\prime}}{I_{\lambda}\left[\left(\alpha_{j}, A_{j}\right)_{1, q} ;\left(\beta_{j}, B_{j}\right)_{1, p}\right] f(z)}\right\}>\mu,
$$

but $\Re\{z\} \leq|z|$ then

$$
\left|\frac{z\left[I_{\lambda}\left[\left(\alpha_{j}, A_{j}\right)_{1, q^{\prime}} ;\left(\beta_{j}, B_{j}\right)_{1, p}\right] f(z)\right]^{\prime}}{I_{\lambda}\left[\left(\alpha_{j}, A_{j}\right)_{1, q^{\prime}} ;\left(\beta_{j}, B_{j}\right)_{1, p}\right] f(z)}\right|>\mu .
$$

By a computation, we obtain (2.1).

Corollary 2.2. Let the function $f(z)$ belong to the class $S_{\lambda}^{\mu}\left[\left(\alpha_{j}, A_{j}\right)_{1, q} ;\left(\beta_{j}, B_{j}\right)_{1, p}\right]$. Then

$$
\left|a_{n}\right| \leq \frac{(1-\mu)}{H_{n-1}\left|\mu(\lambda+1)_{n-1}-\left((\lambda+1)_{n}-(\lambda)_{n}\right)\right|}, \quad 0 \leq \mu<1,
$$

where $H_{n-1}$ is defined in (2.2). 
Theorem 2.3. Let $f(z) \in \mathcal{A}$. Then $f(z) \in C_{\lambda}^{\mu}\left[\left(\alpha_{j}, A_{j}\right)_{1, q} ;\left(\beta_{j}, B_{j}\right)_{1, p}\right]$ if and only if

$$
\sum_{n=2}^{\infty} n H_{n-1}\left|a_{n}\right|\left|\mu(\lambda+1)_{n-1}-\left((\lambda+1)_{n}-(\lambda)_{n}\right)\right| \leq 1-\mu, \quad 0 \leq \mu<1,
$$

where $H_{n-1}$ is defined in (2.2).

Corollary 2.4. Let the function $f(z)$ belong to the class $C_{\lambda}^{\mu}\left[\left(\alpha_{j}, A_{j}\right)_{1, q} ;\left(\beta_{j}, B_{j}\right)_{1, p}\right]$. Then

$$
\left|a_{n}\right| \leq \frac{(1-\mu)}{n H_{n-1}\left|\mu(\lambda+1)_{n-1}-\left((\lambda+1)_{n}-(\lambda)_{n}\right)\right|}, \quad 0 \leq \mu<1,
$$

where $H_{n-1}$ is defined in (2.2).

Theorem 2.5. Let $f(z) \in S_{\lambda}^{\mu}\left[\left(\alpha_{j}, A_{j}\right)_{1, q} ;\left(\beta_{j}, B_{j}\right)_{1, p}\right]$, then

$$
\begin{aligned}
& |f(z)| \geq|z|-\frac{(1-\mu)}{H_{1}\left|\mu(\lambda+1)_{1}-\left((\lambda+1)_{2}-(\lambda)_{2}\right)\right|}|z|^{2}, \\
& |f(z)| \leq|z|+\frac{(1-\mu)}{H_{1}\left|\mu(\lambda+1)_{1}-\left((\lambda+1)_{2}-(\lambda)_{2}\right)\right|}|z|^{2},
\end{aligned}
$$

for $z \in U$ where $H_{n-1}$ is defined in (2.2).

Proof. If $f(z) \in S_{\lambda}^{\mu}\left[\left(\alpha_{j}, A_{j}\right)_{1, q} ;\left(\beta_{j}, B_{j}\right)_{1, p}\right]$ then in view of Theorem 2.1, we have

$$
\begin{aligned}
H_{1}\left|\mu(\lambda+1)_{1}-\left((\lambda+1)_{2}-(\lambda)_{2}\right)\right| \sum_{n=2}^{\infty}\left|a_{n}\right| & \leq \sum_{n=2}^{\infty} H_{n-1}\left|a_{n}\right|\left|\mu(\lambda+1)_{n-1}-\left((\lambda+1)_{n}-(\lambda)_{n}\right)\right| \\
& \leq 1-\mu .
\end{aligned}
$$

This yields

$$
\sum_{n=2}^{\infty}\left|a_{n}\right| \leq \frac{1-\mu}{H_{1}\left|\mu(\lambda+1)_{1}-\left((\lambda+1)_{2}-(\lambda)_{2}\right)\right|}
$$

Now

$$
\begin{aligned}
|f(z)| & \geq|z|-|z|^{2} \sum_{n=2}^{\infty}\left|a_{n}\right| \\
& \geq|z|-\frac{(1-\mu)}{H_{1}\left|\mu(\lambda+1)_{1}-\left((\lambda+1)_{2}-(\lambda)_{2}\right)\right|}|z|^{2} .
\end{aligned}
$$

Also,

$$
|f(z)| \leq|z|+\frac{(1-\mu)}{H_{1}\left|\mu(\lambda+1)_{1}-\left((\lambda+1)_{2}-(\lambda)_{2}\right)\right|}|z|^{2} .
$$

Hence the proof is complete. 
Corollary 2.6. Under the hypothesis of Theorem 2.5, $f(z)$ is included in a disk with its center at the origin and radius $r$ given by

$$
r=1+\frac{(1-\mu)}{H_{1}\left|\mu(\lambda+1)_{1}-\left((\lambda+1)_{2}-(\lambda)_{2}\right)\right|} .
$$

In the same way, we can prove the following result.

Theorem 2.7. Let $f(z) \in C_{\lambda}^{\mu}\left[\left(\alpha_{j}, A_{j}\right)_{1, q} ;\left(\beta_{j}, B_{j}\right)_{1, p}\right]$ then

$$
\begin{aligned}
& |f(z)| \geq|z|-\frac{(1-\mu)}{2 H_{1}\left|\mu(\lambda+1)_{1}-\left((\lambda+1)_{2}-(\lambda)_{2}\right)\right|}|z|^{2}, \\
& |f(z)| \leq|z|+\frac{(1-\mu)}{2 H_{1}\left|\mu(\lambda+1)_{1}-\left((\lambda+1)_{2}-(\lambda)_{2}\right)\right|}|z|^{2},
\end{aligned}
$$

for $z \in U$ where $H_{n-1}$ is defined in (2.2).

Corollary 2.8. Under the hypothesis of Theorem 2.7, $f(z)$ is included in a disk with its center at the origin and radius $r$ given by

$$
r=1+\frac{(1-\mu)}{2 H_{1}\left|\mu(\lambda+1)_{1}-\left((\lambda+1)_{2}-(\lambda)_{2}\right)\right|} .
$$

We next study some properties of the classes $S_{\lambda}^{\mu}\left[\left(\alpha_{j}, A_{j}\right)_{1, q} ;\left(\beta_{j}, B_{j}\right)_{1, p}\right]$ and $C_{\lambda}^{\mu}\left[\left(\alpha_{j}, A_{j}\right)_{1, q}\right.$; $\left.\left(\beta_{j}, B_{j}\right)_{1, p}\right]$.

Theorem 2.9. Let $\lambda>-1$ and $0 \leq \mu_{1}<\mu_{2}<1$. Then

$$
S_{\lambda}^{\mu_{2}}\left[\left(\alpha_{j}, A_{j}\right)_{1, q} ;\left(\beta_{j}, B_{j}\right)_{1, p}\right] \subset S_{\lambda}^{\mu_{1}}\left[\left(\alpha_{j}, A_{j}\right)_{1, q} ;\left(\beta_{j}, B_{j}\right)_{1, p}\right] .
$$

Proof. By using Theorem 2.1.

Theorem 2.10. Let $-1<\lambda_{1} \leq \lambda_{2}$ and $0 \leq \mu<1$. Then

$$
S_{\lambda_{1}}^{\mu}\left[\left(\alpha_{j}, A_{j}\right)_{1, q^{\prime}}\left(\beta_{j}, B_{j}\right)_{1, p}\right] \supseteq S_{\lambda_{2}}^{\mu}\left[\left(\alpha_{j}, A_{j}\right)_{1, q^{\prime}} ;\left(\beta_{j}, B_{j}\right)_{1, p}\right] .
$$

Proof. By using Theorem 2.1.

Theorem 2.11. Let $\lambda>-1$ and $0 \leq \mu_{1}<\mu_{2}<1$. Then

$$
C_{\lambda}^{\mu_{2}}\left[\left(\alpha_{j}, A_{j}\right)_{1, q^{\prime}}\left(\beta_{j}, B_{j}\right)_{1, p}\right] \subset C_{\lambda}^{\mu_{1}}\left[\left(\alpha_{j}, A_{j}\right)_{1, q^{\prime}}\left(\beta_{j}, B_{j}\right)_{1, p}\right] .
$$

Proof. By using Theorem 2.3.

Theorem 2.12. Let $-1<\lambda_{1} \leq \lambda_{2}$ and $0 \leq \mu<1$. Then

$$
C_{\lambda_{1}}^{\mu}\left[\left(\alpha_{j}, A_{j}\right)_{1, q} ;\left(\beta_{j}, B_{j}\right)_{1, p}\right] \supseteq C_{\lambda_{2}}^{\mu}\left[\left(\alpha_{j}, A_{j}\right)_{1, q} ;\left(\beta_{j}, B_{j}\right)_{1, p}\right] .
$$

Proof. By using Theorem 2.3. 


\section{Sandwich results}

By making use of Lemmas 1.6 and 1.7, we prove the following subordination and superordination results.

Theorem 3.1. Let $q(z) \neq 0$ be univalent in $U$ such that $z q^{\prime}(z) / q(z)$ is starlike univalent in $U$ and

$$
\Re\left\{1+\frac{\alpha}{\gamma} q(z)+\frac{z q^{\prime \prime}(z)}{q^{\prime}(z)}-\frac{z q^{\prime}(z)}{q(z)}\right\}>0, \quad \alpha, \gamma \in \mathbb{C}, \gamma \neq 0
$$

If $f \in \mathcal{A}$ satisfies the subordination

$$
\begin{aligned}
& \alpha\left\{\frac{z\left[I_{\lambda}\left[\left(\alpha_{j}, A_{j}\right)_{1, q^{\prime}}\left(\beta_{j}, B_{j}\right)_{1, p}\right] f(z)\right]^{\prime}}{\Phi\left[I_{\lambda}\left[\left(\alpha_{j}, A_{j}\right)_{1, q^{\prime}}\left(\beta_{j}, B_{j}\right)_{1, p}\right] f(z)\right]}\right\} \\
& \quad+\gamma\left\{1+\frac{z\left[I_{\lambda}\left[\left(\alpha_{j}, A_{j}\right)_{1, q^{\prime}} ;\left(\beta_{j}, B_{j}\right)_{1, p}\right] f(z)\right]^{\prime \prime}}{\left[I_{\lambda}\left[\left(\alpha_{j}, A_{j}\right)_{1, q^{\prime}}\left(\beta_{j}, B_{j}\right)_{1, p}\right] f(z)\right]^{\prime}}-\frac{z \Phi^{\prime}\left[I_{\lambda}\left[\left(\alpha_{j}, A_{j}\right)_{1, q^{\prime}}\left(\beta_{j}, B_{j}\right)_{1, p}\right] f(z)\right]}{\Phi\left[I_{\lambda}\left[\left(\alpha_{j}, A_{j}\right)_{1, q^{\prime}}\left(\beta_{j}, B_{j}\right)_{1, p}\right] f(z)\right]}\right\} \\
& \quad \prec \alpha q(z)+\frac{\gamma z q^{\prime}(z)}{q(z)},
\end{aligned}
$$

then

$$
\frac{z\left[I_{\lambda}\left[\left(\alpha_{j}, A_{j}\right)_{1, q^{\prime}}\left(\beta_{j}, B_{j}\right)_{1, p}\right] f(z)\right]^{\prime}}{\Phi\left[I_{\lambda}\left[\left(\alpha_{j}, A_{j}\right)_{1, q^{\prime}}\left(\beta_{j}, B_{j}\right)_{1, p}\right] f(z)\right]} \prec q(z)
$$

and $q(z)$ is the best dominant.

Proof. Our aim is to apply Lemma 1.6. Setting

$$
p(z):=\frac{z\left[I_{\lambda}\left[\left(\alpha_{j}, A_{j}\right)_{1, q^{\prime}} ;\left(\beta_{j}, B_{j}\right)_{1, p}\right] f(z)\right]^{\prime}}{\Phi\left[I_{\lambda}\left[\left(\alpha_{j}, A_{j}\right)_{1, q} ;\left(\beta_{j}, B_{j}\right)_{1, p}\right] f(z)\right]}
$$

Computation shows that

$$
\frac{z p^{\prime}(z)}{p(z)}=1+\frac{z\left[I_{\lambda}\left[\left(\alpha_{j}, A_{j}\right)_{1, q^{\prime}} ;\left(\beta_{j}, B_{j}\right)_{1, p}\right] f(z)\right]^{\prime \prime}}{\left[I_{\lambda}\left[\left(\alpha_{j}, A_{j}\right)_{1, q^{\prime}} ;\left(\beta_{j}, B_{j}\right)_{1, p}\right] f(z)\right]^{\prime}}-\frac{z \Phi^{\prime}\left[I_{\lambda}\left[\left(\alpha_{j}, A_{j}\right)_{1, q^{\prime}}\left(\beta_{j}, B_{j}\right)_{1, p}\right] f(z)\right]}{\Phi\left[I_{\curlywedge}\left[\left(\alpha_{j}, A_{j}\right)_{1, q^{\prime}}\left(\beta_{j}, B_{j}\right)_{1, p}\right] f(z)\right]}
$$

which yields the following subordination:

$$
\alpha p(z)+\frac{\gamma z p^{\prime}(z)}{p(z)} \prec \alpha q(z)+\frac{\gamma z q^{\prime}(z)}{q(z)}, \quad \alpha, \gamma \in \mathbb{C}
$$


By setting

$$
\theta(\omega):=\alpha \omega, \quad \phi(\omega):=\frac{\gamma}{\omega}, \quad \gamma \neq 0,
$$

it can be easily observed that $\theta(\omega)$ is analytic in $\mathbb{C}$ and $\phi(\omega)$ is analytic in $\mathbb{C} \backslash\{0\}$ and that $\phi(\omega) \neq 0$ when $\omega \in \mathbb{C} \backslash\{0\}$. Also, by letting

$$
\begin{gathered}
Q(z)=z q^{\prime}(z) \phi(q(z))=\gamma z \frac{q^{\prime}(z)}{q(z)} \\
h(z)=\theta(q(z))+Q(z)=\alpha q(z)+\gamma z \frac{q^{\prime}(z)}{q(z)},
\end{gathered}
$$

we find that $Q(z)$ is starlike univalent in $U$ and that

$$
\mathfrak{R}\left\{\frac{z h^{\prime}(z)}{Q(z)}\right\}=\left\{1+\frac{\alpha}{\gamma} q(z)+\frac{z q^{\prime \prime}(z)}{q^{\prime}(z)}-\frac{z q^{\prime}(z)}{q(z)}\right\}>0 .
$$

Then the relation (3.3) follows by an application of Lemma 1.6.

Corollary 3.2. Let the assumptions of Theorem 2.1 hold. Then the subordination

$$
\begin{aligned}
& (\alpha-\gamma)\left\{\frac{z\left[I_{\lambda}\left[\left(\alpha_{j}, A_{j}\right)_{1, q^{\prime}}\left(\beta_{j}, B_{j}\right)_{1, p}\right] f(z)\right]^{\prime}}{\left[I_{\lambda}\left[\left(\alpha_{j}, A_{j}\right)_{1, q^{\prime}} ;\left(\beta_{j}, B_{j}\right)_{1, p}\right] f(z)\right]}\right\}+\gamma\left\{1+\frac{z\left[I_{\lambda}\left[\left(\alpha_{j}, A_{j}\right)_{1, q^{\prime}}\left(\beta_{j}, B_{j}\right)_{1, p}\right] f(z)\right]^{\prime \prime}}{\left[I_{\lambda}\left[\left(\alpha_{j}, A_{j}\right)_{1, q^{\prime}}\left(\beta_{j}, B_{j}\right)_{1, p}\right] f(z)\right]^{\prime}}\right\} \\
& \quad \prec \alpha q(z)+\frac{\gamma z q^{\prime}(z)}{q(z)},
\end{aligned}
$$

implies

$$
\frac{z\left[I_{\lambda}\left[\left(\alpha_{j}, A_{j}\right)_{1, q} ;\left(\beta_{j}, B_{j}\right)_{1, p}\right] f(z)\right]^{\prime}}{\left[I_{\curlywedge}\left[\left(\alpha_{j}, A_{j}\right)_{1, q} ;\left(\beta_{j}, B_{j}\right)_{1, p}\right] f(z)\right]} \prec q(z)
$$

and $q(z)$ is the best dominant.

Proof. By letting $\Phi(\omega):=\omega$.

Corollary 3.3. If $f \in \mathcal{A}$ and assume that (3.1) holds then

$$
1+\frac{z\left[I_{\lambda}\left[\left(\alpha_{j}, A_{j}\right)_{1, q^{\prime}}\left(\beta_{j}, B_{j}\right)_{1, p}\right] f(z)\right]^{\prime \prime}}{\left[I_{\lambda}\left[\left(\alpha_{j}, A_{j}\right)_{1, q^{\prime}}\left(\beta_{j}, B_{j}\right)_{1, p}\right] f(z)\right]^{\prime}} \prec \frac{1+A z}{1+B z}+\frac{(A-B) z}{(1+A z)(1+B z)}
$$

implies

$$
\frac{z\left[I_{\lambda}\left[\left(\alpha_{j}, A_{j}\right)_{1, q^{\prime}} ;\left(\beta_{j}, B_{j}\right)_{1, p}\right] f(z)\right]^{\prime}}{\left[I_{\lambda}\left[\left(\alpha_{j}, A_{j}\right)_{1, q^{\prime}}\left(\beta_{j}, B_{j}\right)_{1, p}\right] f(z)\right]} \prec \frac{1+A z}{1+B z}, \quad-1 \leq B<A \leq 1,
$$

and $(1+A z) /(1+B z)$ is the best dominant. 
Proof. By setting $\Phi(\omega):=\omega, \alpha=\gamma=1$, and $q(z):=(1+A z) /(1+B z)$, where $-1 \leq B<A \leq 1$.

Corollary 3.4. If $f \in \mathcal{A}$ and assume that (3.1) holds then

$$
1+\frac{z\left[I_{\lambda}\left[\left(\alpha_{j}, A_{j}\right)_{1, q^{\prime}} ;\left(\beta_{j}, B_{j}\right)_{1, p}\right] f(z)\right]^{\prime \prime}}{\left[I_{\lambda}\left[\left(\alpha_{j}, A_{j}\right)_{1, q^{\prime}} ;\left(\beta_{j}, B_{j}\right)_{1, p}\right] f(z)\right]^{\prime}} \prec \frac{1+z}{1-z}+\frac{2 z}{1-z^{2}}
$$

implies

$$
\frac{z\left[I_{\curlywedge}\left[\left(\alpha_{j}, A_{j}\right)_{1, q^{\prime}}\left(\beta_{j}, B_{j}\right)_{1, p}\right] f(z)\right]^{\prime}}{I_{\lambda}\left[\left(\alpha_{j}, A_{j}\right)_{1, q^{\prime}}\left(\beta_{j}, B_{j}\right)_{1, p}\right] f(z)} \prec \frac{1+z}{1-z^{\prime}}
$$

and $(1+z) /(1-z)$ is the best dominant.

Proof. By setting $\Phi(\omega):=\omega, \alpha=\gamma=1$, and $q(z):=(1+z) /(1-z)$.

Corollary 3.5. If $f \in \mathcal{A}$ and assume that (3.1) holds then

$$
1+\frac{z\left[I_{\lambda}\left[\left(\alpha_{j}, A_{j}\right)_{1, q} ;\left(\beta_{j}, B_{j}\right)_{1, p}\right] f(z)\right]^{\prime \prime}}{\left[I_{\curlywedge}\left[\left(\alpha_{j}, A_{j}\right)_{1, q} ;\left(\beta_{j}, B_{j}\right)_{1, p}\right] f(z)\right]^{\prime}} \prec e^{A z}+A z
$$

implies

$$
\frac{z\left[I_{\lambda}\left[\left(\alpha_{j}, A_{j}\right)_{1, q} ;\left(\beta_{j}, B_{j}\right)_{1, p}\right] f(z)\right]^{\prime}}{I_{\lambda}\left[\left(\alpha_{j}, A_{j}\right)_{1, q} ;\left(\beta_{j}, B_{j}\right)_{1, p}\right] f(z)} \prec e^{A z},
$$

and $e^{A z}$ is the best dominant.

Proof. By setting $\Phi(\omega):=\omega, \alpha=\gamma=1$, and $q(z):=e^{A z},|A|<\pi$.

Theorem 3.6. Let $q(z) \neq 0$ be convex univalent in the unit disk $U$. Suppose that

$$
\mathfrak{R}\left\{\frac{\alpha}{\gamma} q(z)\right\}>0, \quad \alpha, \gamma \in \mathbb{C} \text { for } z \in U,
$$

and that $z q^{\prime}(z) / q(z)$ is starlike univalent in $U$. If $z\left[I_{\lambda}\left[\left(\alpha_{j}, A_{j}\right)_{1, q} ;\left(\beta_{j}, B_{j}\right)_{1, p}\right] f(z)\right]^{\prime} / \Phi\left[I_{\lambda}\left[\left(\alpha_{j}\right.\right.\right.$, $\left.\left.\left.A_{j}\right)_{1, q} ;\left(\beta_{j}, B_{j}\right)_{1, p}\right] f(z)\right] \in \mathscr{H}[q(0), 1] \cap Q$ where $f \in \mathcal{A}$,

$$
\begin{aligned}
& \alpha\left\{\frac{z\left[I_{\lambda}\left[\left(\alpha_{j}, A_{j}\right)_{1, q} ;\left(\beta_{j}, B_{j}\right)_{1, p}\right] f(z)\right]^{\prime}}{\Phi\left[I_{\lambda}\left[\left(\alpha_{j}, A_{j}\right)_{1, q^{\prime}}\left(\beta_{j}, B_{j}\right)_{1, p}\right] f(z)\right]}\right\} \\
& \quad+\gamma\left\{1+\frac{z\left[I_{\lambda}\left[\left(\alpha_{j}, A_{j}\right)_{1, q^{\prime}} ;\left(\beta_{j}, B_{j}\right)_{1, p}\right] f(z)\right]^{\prime \prime}}{\left[I_{\lambda}\left[\left(\alpha_{j}, A_{j}\right)_{1, q^{\prime}}\left(\beta_{j}, B_{j}\right)_{1, p}\right] f(z)\right]^{\prime}}-\frac{z \Phi^{\prime}\left[I_{\lambda}\left[\left(\alpha_{j}, A_{j}\right)_{1, q^{\prime}}\left(\beta_{j}, B_{j}\right)_{1, p}\right] f(z)\right]}{\Phi\left[I_{\lambda}\left[\left(\alpha_{j}, A_{j}\right)_{1, q} ;\left(\beta_{j}, B_{j}\right)_{1, p}\right] f(z)\right]}\right\}
\end{aligned}
$$


is univalent in $U$ and the subordination

$$
\begin{aligned}
& \alpha q(z)+\frac{\gamma z q^{\prime}(z)}{q(z)}< \alpha\left\{\frac{z\left[I_{\lambda}\left[\left(\alpha_{j}, A_{j}\right)_{1, q} ;\left(\beta_{j}, B_{j}\right)_{1, p}\right] f(z)\right]^{\prime}}{\Phi\left[I_{\lambda}\left[\left(\alpha_{j}, A_{j}\right)_{1, q^{\prime}}\left(\beta_{j}, B_{j}\right)_{1, p}\right] f(z)\right]}\right\} \\
&+\gamma\left\{1+\frac{z\left[I_{\lambda}\left[\left(\alpha_{j}, A_{j}\right)_{1, q} ;\left(\beta_{j}, B_{j}\right)_{1, p}\right] f(z)\right]^{\prime \prime}}{\left[I_{\lambda}\left[\left(\alpha_{j}, A_{j}\right)_{1, q} ;\left(\beta_{j}, B_{j}\right)_{1, p}\right] f(z)\right]^{\prime}}\right. \\
& \\
&\left.\quad-\frac{z \Phi^{\prime}\left[I_{\lambda}\left[\left(\alpha_{j}, A_{j}\right)_{1, q} ;\left(\beta_{j}, B_{j}\right)_{1, p}\right] f(z)\right]}{\Phi\left[I_{\lambda}\left[\left(\alpha_{j}, A_{j}\right)_{1, q} ;\left(\beta_{j}, B_{j}\right)_{1, p}\right] f(z)\right]}\right\}
\end{aligned}
$$

holds, then

$$
q(z) \prec \frac{z\left[I_{\lambda}\left[\left(\alpha_{j}, A_{j}\right)_{1, q} ;\left(\beta_{j}, B_{j}\right)_{1, p}\right] f(z)\right]^{\prime}}{\Phi\left[I_{\lambda}\left[\left(\alpha_{j}, A_{j}\right)_{1, q} ;\left(\beta_{j}, B_{j}\right)_{1, p}\right] f(z)\right]}
$$

and $q$ is the best subordinant.

Proof. Our aim is to apply Lemma 1.7. Setting

$$
p(z):=\frac{z\left[I_{\lambda}\left[\left(\alpha_{j}, A_{j}\right)_{1, q^{\prime}}\left(\beta_{j}, B_{j}\right)_{1, p}\right] f(z)\right]^{\prime}}{\Phi\left[I_{\lambda}\left[\left(\alpha_{j}, A_{j}\right)_{1, q} ;\left(\beta_{j}, B_{j}\right)_{1, p}\right] f(z)\right]}
$$

Computation shows that

$$
\frac{z p^{\prime}(z)}{p(z)}=1+\frac{z\left[I_{\lambda}\left[\left(\alpha_{j}, A_{j}\right)_{1, q^{\prime}}\left(\beta_{j}, B_{j}\right)_{1, p}\right] f(z)\right]^{\prime \prime}}{\left[I_{\lambda}\left[\left(\alpha_{j}, A_{j}\right)_{1, q^{\prime}}\left(\beta_{j}, B_{j}\right)_{1, p}\right] f(z)\right]^{\prime}}-\frac{z \Phi^{\prime}\left[I_{\lambda}\left[\left(\alpha_{j}, A_{j}\right)_{1, q^{\prime}} ;\left(\beta_{j}, B_{j}\right)_{1, p}\right] f(z)\right]}{\Phi\left[I_{\lambda}\left[\left(\alpha_{j}, A_{j}\right)_{1, q^{\prime}}\left(\beta_{j}, B_{j}\right)_{1, p}\right] f(z)\right]},
$$

which yields the following subordination

$$
\alpha q(z)+\frac{\gamma z q^{\prime}(z)}{q(z)} \prec \alpha p(z)+\frac{\gamma z p^{\prime}(z)}{p(z)}, \quad \alpha, \gamma \in \mathbb{C}
$$

By setting

$$
\vartheta(\omega):=\alpha \omega, \quad \varphi(\omega):=\frac{\gamma}{\omega}, \quad \gamma \neq 0
$$

it can be easily observed that $\theta(\omega)$ is analytic in $\mathbb{C}$ and $\phi(\omega)$ is analytic in $\mathbb{C} \backslash\{0\}$, and that $\phi(\omega) \neq 0$ when $\omega \in \mathbb{C} \backslash\{0\}$. Also, we obtain

$$
\Re\left\{\frac{\vartheta^{\prime}(q(z))}{\varphi(q(z))}\right\}=\Re\left\{\frac{\alpha}{\gamma} q(z)\right\}>0 .
$$

Then (3.21) follows by an application of Lemma 1.7.

Combining Theorems 3.1 and 3.6 in order to get the following sandwich theorems 
Theorem 3.7. Let $q_{1}(z) \neq 0, q_{2}(z) \neq 0$ be convex univalent in the unit disk $U$ satisfying (3.18) and (3.1), respectively. Suppose that $z q_{i}^{\prime}(z) / q_{i}(z), i=1,2$, is starlike univalent in $U$. If

$$
\frac{z\left[I_{\lambda}\left[\left(\alpha_{j}, A_{j}\right)_{1, q^{\prime}} ;\left(\beta_{j}, B_{j}\right)_{1, p}\right] f(z)\right]^{\prime}}{\Phi\left[I_{\lambda}\left[\left(\alpha_{j}, A_{j}\right)_{1, q} ;\left(\beta_{j}, B_{j}\right)_{1, p}\right] f(z)\right]} \in \mathscr{H}[q(0), 1] \cap Q,
$$

where $f \in \mathcal{A}$,

$$
\begin{aligned}
& \alpha\left\{\frac{z\left[I_{\lambda}\left[\left(\alpha_{j}, A_{j}\right)_{1, q^{\prime}} ;\left(\beta_{j}, B_{j}\right)_{1, p}\right] f(z)\right]^{\prime}}{\Phi\left[I_{\lambda}\left[\left(\alpha_{j}, A_{j}\right)_{1, q^{\prime}}\left(\beta_{j}, B_{j}\right)_{1, p}\right] f(z)\right]}\right\} \\
& \quad+\gamma\left\{1+\frac{z\left[I_{\curlywedge}\left[\left(\alpha_{j}, A_{j}\right)_{1, q^{\prime}}\left(\beta_{j}, B_{j}\right)_{1, p}\right] f(z)\right]^{\prime \prime}}{\left[I_{\lambda}\left[\left(\alpha_{j}, A_{j}\right)_{1, q^{\prime}} ;\left(\beta_{j}, B_{j}\right)_{1, p}\right] f(z)\right]^{\prime}}-\frac{z \Phi^{\prime}\left[I_{\curlywedge}\left[\left(\alpha_{j}, A_{j}\right)_{1, q^{\prime}}\left(\beta_{j}, B_{j}\right)_{1, p}\right] f(z)\right]}{\Phi\left[I_{\lambda}\left[\left(\alpha_{j}, A_{j}\right)_{1, q^{\prime}}\left(\beta_{j}, B_{j}\right)_{1, p}\right] f(z)\right]}\right\}
\end{aligned}
$$

is univalent in $U$, and the subordination

$$
\begin{gathered}
\alpha q_{1}(z)+\frac{\gamma z q_{1}^{\prime}(z)}{q_{1}(z)} \prec \alpha\left\{\frac{z\left[I_{\lambda}\left[\left(\alpha_{j}, A_{j}\right)_{1, q^{\prime}}\left(\beta_{j}, B_{j}\right)_{1, p}\right] f(z)\right]^{\prime}}{\Phi\left[I_{\lambda}\left[\left(\alpha_{j}, A_{j}\right)_{1, q} ;\left(\beta_{j}, B_{j}\right)_{1, p}\right] f(z)\right]}\right\} \\
+\gamma\left\{1+\frac{z\left[I_{\lambda}\left[\left(\alpha_{j}, A_{j}\right)_{1, q^{\prime}}\left(\beta_{j}, B_{j}\right)_{1, p}\right] f(z)\right]^{\prime \prime}}{\left[I_{\lambda}\left[\left(\alpha_{j}, A_{j}\right)_{1, q^{\prime}}\left(\beta_{j}, B_{j}\right)_{1, p}\right] f(z)\right]^{\prime}}\right. \\
\left.\quad-\frac{z \Phi^{\prime}\left[I_{\lambda}\left[\left(\alpha_{j}, A_{j}\right)_{1, q^{\prime}}\left(\beta_{j}, B_{j}\right)_{1, p}\right] f(z)\right]}{\Phi\left[I_{\lambda}\left[\left(\alpha_{j}, A_{j}\right)_{1, q^{\prime}}\left(\beta_{j}, B_{j}\right)_{1, p}\right] f(z)\right]}\right\} \\
<\alpha q_{2}(z)+\frac{\gamma z q_{2}^{\prime}(z)}{q_{2}(z)}
\end{gathered}
$$

holds, then

$$
q_{1}(z) \prec \frac{z\left[I_{\curlywedge}\left[\left(\alpha_{j}, A_{j}\right)_{1, q} ;\left(\beta_{j}, B_{j}\right)_{1, p}\right] f(z)\right]^{\prime}}{\Phi\left[I_{\curlywedge}\left[\left(\alpha_{j}, A_{j}\right)_{1, q^{\prime}} ;\left(\beta_{j}, B_{j}\right)_{1, p}\right] f(z)\right]} \prec q_{2}(z),
$$

and $q_{1}(z)$ is the best subordinant and $q_{2}(z)$ is the best dominant.

\section{Acknowledgment}

The work presented here was supported by SAGA: STGL-012-2006, Academy of Sciences, Malaysia.

\section{References}

[1] K. I. Noor, “On new classes of integral operators," Journal of Natural Geometry, vol. 16, no. 1-2, pp. 71-80, 1999.

[2] K. I. Noor and M. A. Noor, "On integral operators," Journal of Mathematical Analysis and Applications, vol. 238, no. 2, pp. 341-352, 1999. 
[3] C. Fox, "The asymptotic expansion of the generalized hypergeometric function," Proceedings of the London Mathematical Society, vol. 27, no. 1, pp. 389-400, 1928.

[4] E. M. Wright, "The asymptotic expansion of the generalized hypergeometric function," Journal of the London Mathematical Society, vol. 10, no. 40, pp. 286-293, 1935.

[5] E. M. Wright, "The asymptotic expansion of the generalized hypergeometric function," Proceedings of the London Mathematical Society, vol. 46, no. 1, pp. 389-408, 1940.

[6] L. Brickman, "Ф-like analytic functions. I," Bulletin of the American Mathematical Society, vol. 79, no. 3, pp. 555-558, 1973.

[7] S. S. Miller and P. T. Mocanu, "Subordinants of differential superordinations," Complex Variables and Elliptic Equations, vol. 48, no. 10, pp. 815-826, 2003.

[8] S. S. Miller and P. T. Mocanu, Differential Subordinations: Theory and Application, vol. 225 of Monographs and Textbooks in Pure and Applied Mathematics, Marcel Dekker, New York, NY, USA, 2000.

[9] T. Bulboacă, "Classes of first-order differential superordinations," Demonstratio Mathematica, vol. 35, no. 2, pp. 287-292, 2002. 